pleurodesis for primary spontaneous pneumothorax. Eur Respir J 2007; 29: 757-760.

2 Tschopp JM, Brutsche M, Frey JG. Treatment of complicated spontaneous pneumothorax by simple talc pleurodesis under thoracoscopy and local anaesthesia. Thorax 1997; 52: 329-332.

3 Vanderschueren RG. [Pleural talcage in patients with spontaneous pneumothorax (author's transl.).] Poumon Coeur 1981; 37: 273-276.

4 Lange P, Mortensen J, Groth S. Lung function 22-35 years after treatment of idiopathic spontaneous pneumothorax with talc poudrage or simple drainage. Thorax 1988; 43: 559-561.
5 Schoenenberger RA, Haefeli WE, Weiss P, Ritz R. Evaluation of conventional chest tube therapy for iatrogenic pneumothorax. Chest 1993; 104: 1770-1772.

6 Abolnik IZ, Lossos IS, Gillis D, Breuer R. Primary spontaneous pneumothorax in men. Am J Med Sci 1993; 305: 297-303.

7 Ben-Nun A, Soudack M, Best LA. Video-assisted thoracoscopic surgery for recurrent spontaneous pneumothorax: the long-term benefit. World J Surg 2006; 30: 285-290.

8 Noppen M. Management of primary spontaneous pneumothorax. Curr Opin Pulm Med 2003; 9: 272-275.

DOI: $10.1183 / 09031936.00065407$

\title{
Mitochondrial dysfunction in COPD patients with low body mass index
}

\section{To the Editors:}

The potential importance of mitochondrial (dys)function in common chronic diseases is increasingly recognised [1]. Accordingly, we read with great interest the article by RABINOVICH et al. [2], which was recently published in the European Respiratory Journal. RABINOVICH et al. [2] showed that chronic obstructive pulmonary disease (COPD) patients with a low body mass index (BMI; $19.2 \pm 0.6 \mathrm{~kg} \cdot \mathrm{m}^{-2}$ ) have a dysfunctional mitochondrial electron transport chain in comparison with COPD patients with a "normal" BMI $\left(29.0 \pm 1.7 \mathrm{~kg} \cdot \mathrm{m}^{-2}\right)$ and healthy controls $\left(27.9 \pm 1.9 \mathrm{~kg} \cdot \mathrm{m}^{-2}\right)$. The function of the mitochondrial electron transport chain was assessed as acceptor control ratio (i.e. the efficiency of oxidative phosphorylation) in freshly obtained muscle biopsies.

We recently showed that cigarette smoke, which is the major cause of COPD in the Western world, can induce significant mitochondrial dysfunction [3]. This dysfunction was the consequence of a cigarette smoke-induced blockage of the mitochondrial electron transport chain, resulting in a decreased mitochondrial respiration rate and a corresponding decrease in adenosine triphosphate production.

In the study performed by RABINOVICH et al. [2], the prevalence of current and past smoking was not reported. As a consequence, the potential influence of smoking on the outcome of the study was neither analysed nor discussed. Due to our recent observations, and reports from other groups about the induction of mitochondrial dysfunction by cigarette smoke even in circulating cells that do not reside in pulmonary tissues [4], we thought it would be very interesting to know how the results of the clinical study performed by RABINOVICH et al. [2] would appear if current (and past) smoking were taken into account. Furthermore, it would be interesting to know how the significantly lower acceptor control ratio results reported in their study affect the principal function of mitochondria: adenosine triphosphate production.

\author{
D-J. Slebos*, M. van der Toorn", S.J.L. Bakker and H.F. \\ Kauffman ${ }^{+}$ \\ Depts of *Pulmonary Diseases, "Internal Medicine, and \\ ${ }^{+}$Pathology, and "Laboratory of Allergology and Pulmonary \\ Diseases, University Medical Center Groningen, University of \\ Groningen, Groningen, The Netherlands.
}

\section{STATEMENT OF INTEREST}

None declared.

\section{REFERENCES}

1 Lane N. Mitochondrial disease: powerhouse of disease. Nature 2006; 440: 600-602.

2 Rabinovich RA, Bastos R, Ardite E, et al. Mitochondrial dysfunction in COPD patients with low body mass index. Eur Respir J 2007; 29: 643-650.

3 van der Toorn M, Slebos DJ, de Bruin HG, et al. Cigarette smoke induced blockade of the mitochondrial respiratory chain switches lung epithelial cell apoptosis into necrosis. Am J Physiol Lung Cell Mol Physiol 2007; 292: L1211-L1218.

4 Miro O, Alonso JR, Jarreta D, Casademont J, UrbanoMarquez A, Cardellach F. Smoking disturbs mitochondrial respiratory chain function and enhances lipid peroxidation on human circulating lymphocytes. Carcinogenesis 1999; 20: 1331-1336.

DOI: $10.1183 / 09031936.00047907$

\section{From the authors:}

We would like to thank D-J. Slebos and co-workers for their interesting and assertive contribution to the present issue of the European Respiratory Journal. Following their arguments, one can reasonably state that because tobacco smoking is the main cause of chronic obstructive pulmonary disease (COPD), it might also play a role in a mechanism leading to the 
extra-pulmonary effects of the disease. In their recently published work [1], it was shown that cigarette smoke condensate can induce mitochondrial dysfunction in an in vitro model. This makes it more tempting to embrace their hypothesis. Nevertheless, one should be cautious in transferring in vitro results to the real situation in patients. We should bear in mind that muscle dysfunction, as one of the so-called systemic effects of COPD, is a complex phenomenon caused by multiple factors, such as oxidative stress, systemic inflammation, muscle disuse and hypoxaemia, among others. Our report on mitochondrial dysfunction at the peripheral muscle level in patients with COPD and low body mass index (and significantly reduced fat-free mass index) [2] should be added to the list of pathological findings at the muscle level in these patients. With regard to the smoking status, there were no differences among the three subpopulations included in our study. None of the subjects smoked at the time of their inclusion in the study. Unfortunately, we do not have data on the accumulative smoking history in this population, which may have helped to shed light on this issue. It should be added that, although causality cannot be established, the significant association between acceptor control ratio and arterial oxygen tension may indicate an abnormal mitochondrial adaptation to long-term repeated episodes of cell hypoxia.

D-J. Slebos and co-workers were also interested in mitochondrial adenosine triphosphate production in our population. Although we did not specifically assess the rate of adenosine triphosphate production, the lower State 3 of respiration in energised mitochondria by succinate in the subpopulation of chronic obstructive pulmonary disease patients with low body mass index reflects a lower oxidative phosphorylation in this subset of patients.

R.A. Rabinovich*, R. Bastos*, E. Ardite*, L. Llinàs*, M. Orozco-Levi ${ }^{\#, \mp}$, J. Gea ${ }^{\#, \text {, J. Vilaró }}{ }^{+}$, J.A. Barberà*, R. Rodriguez-Roisin*, J.C. Fernandez-Checa ${ }^{\S}$ and J. Roca* *Pulmonary Dept (ICT), Hospital Clinic, IDIBAPS, and ${ }^{\S}$ Liver Unit (IMD)-CSIC, IDIBAPS, Universitat de Barcelona, "Muscle and Respiratory System Research Unit, IMIM, CEXS, Universitat Pompeu Fabra, "Respiratory Medicine Dept, Hospital del Mar, and ${ }^{+}$EUIF Blanquerna, Universitat Ramon Llull, Barcelona, Spain.

\section{STATEMENT OF INTEREST}

None declared.

\section{REFERENCES}

1 van der Toorn M, Slebos DJ, de Bruin HG, et al. Cigarette smoke-induced blockade of the mitochondrial respiratory chain switches lung epithelial cell apoptosis into necrosis. Am J Physiol Lung Cell Mol Physiol 2007; 292: L1211-L1218.

2 Rabinovich RA, Bastos R, Ardite E, et al. Mitochondrial dysfunction in COPD patients with low body mass index. Eur Respir J 2007; 29: 643-650.

DOI: $10.1183 / 09031936.00055807$

\section{Is endobronchial ultrasound additive to routine bronchoscopy in diagnosing sarcoidosis?}

To the Editors:

In the June issue of the European Respiratory Journal, WoNG et al. [1] describe the utility of endobronchial ultrasound-guided transbronchial needle aspiration (EBUS-TBNA) in a cohort of 65 patients with suspected sarcoidosis. EBUS-TBNA correctly identified noncaseating granuloma in 56 out of 61 patients, who were ultimately diagnosed with sarcoidosis. Thus, the authors' pre-test criteria for sarcoid were correct in 61 (94\%) out of 65 patients. Some readers may therefore argue that EBUS-TBNA was not required if sarcoidosis can be so accurately identified by a combination of clinical and radiographical information. While the authors clearly excluded patients with "suspected or known malignancy", it is nonetheless surprising that no other causes of mediastinal lymphadenopathy, such as lymphoma, metastatic cancer or infection, were identified in this cohort. In fact, in clinical practice, the reason for performing EBUS-TBNA in patients with diffuse mediastinal lymphadenopathy is to rule out malignancy and infection, as it is often difficult to distinguish stage 1 sarcoidosis from, for instance, lymphoma or granulomatous infection. This important study highlights the diagnostic yield of EBUS-TBNA in an inordinately high-prevalence sarcoidosis population but leaves open the question of general applicability

A prospective study is warranted, investigating the added benefit of endobronchial ultrasound-guided transbronchial needle aspiration to routine bronchoscopy with transbronchial lung biopsy in a more heterogeneous population of patients with idiopathic mediastinal lymphadenopathy.

\section{M.P. Kennedy, C.A. Jimenez, R.C. Morice and G.A. Eapen} Dept of Pulmonary Medicine, University of Texas MD Anderson Cancer Center, Houston, TX, USA.

\section{STATEMENT OF INTEREST}

None declared. 